\title{
A Toolkit for Remote Laboratory Design \& Development
}

\author{
http://dx.doi.org/10.3991/ijoe.v8i1.1874 \\ E. D. Lindsay ${ }^{1}$, S Murray ${ }^{2}$ and B. D. Stumpers ${ }^{1}$ \\ ${ }^{1}$ Curtin University, Perth, Australia \\ ${ }^{2}$ University of Technology, Sydney, Australia
}

\begin{abstract}
Remote laboratories are an increasingly prevalent instructional tool for undergraduate engineering laboratory classes. This increased prevalence brings with it a need to change the model of how remote laboratories are developed. The earlier remote laboratories were developed by individual academics combining their discipline-specific skills with their own ability to implement remote operation. This "cottage industry" model allows for significant local innovation; however it does not support widespread or sustainable implementation of remote laboratories. In order to make remote laboratories a mainstream technology, it is essential that potential academic users are well informed and well supported in considering remote laboratories. There are some well-developed and well-established systems for controlling equipment remotely; what has been missing has been the organizational scaffolding to facilitate the engagement of academics. This paper reports on a resource kit developed by the Australian Labshare project that provides this assistance. This resource kit is intended to provide academics with the resources, information and tools that they need to get started with remote laboratories - building them, using them, and understanding their educational outcomes.
\end{abstract}

Index Terms-Development Resource Kit, Labshare, Remote Laboratories.

\section{INTRODUCTION}

In recent years remote laboratories have transitioned into a maturing technology [1-4]. The benefits of distance education have been documented and reviewed and the specific role of remote laboratories in engineering education has been clearly defined.

While remote labs were originally presented as the second best option to a hands-on lab [5] it has since been shown that each access mode provides a different set of learning outcomes [6]. Additionally, remote laboratories have been shown to provide significant benefits compared to traditional hands on laboratories. Examples include increased time for student access to equipment, resource sharing between institutions to offset costs and a more versatile range of experimentation due to the mitigation of safety issues [7].

The focus of remote laboratory development is now moving towards more sustainable models. Rather than individual academics custom building equipment for their specialized subjects, remote laboratory development is increasingly being carried out by multi-institution consortia, such as iLabs [8], LiLa [3], VISIR [2] and Labshare [4]. These groups allow academics considering remote laboratories to take advantage of pre-existing tools to im- plement their experiments, rather than having to begin from scratch.

Numerous papers have attempted to introduce a defining standard in fields ranging from system architecture to software development. The most successful of these have occurred due to the combined efforts of several institutions. One of the goals of the Australian Labshare project [4] is to implement a similar strategy, while also confronting a number of other challenges such as the pooling of resources, the issue of accreditation as it pertains to courses involving remote laboratories and making remote laboratories more readily available to more than specialized faculty.

In order to inform interested faculty about remote laboratories and to facilitate their involvement in the Labshare project, the Labshare toolkit was developed. The toolkit offers materials in support of remote laboratory technical development, pedagogical efficacy and organizational sustainability. This paper concentrates on the pedagogically-oriented components, which are freely accessible via the Labshare website (http://www.labshare.edu.au/) and currently include:

- The Labshare FAQ

- A Glossary of terms

- A Literature Review of remote laboratories, including an endnote library containing over 380 remote laboratory references and a ranking of the most cited papers

- A framework for determining rig suitability

- A snapshot of the current catalogue of available experiments

- An Accreditation Commentary that details how remote laboratories can contribute to meeting the ABET and EA accreditation criteria

- Sample lesson plans for the available rigs, including templates for layout

- Sample evaluation questions, covering both the development and implementation processes as well as the student usage experience

With access to the toolkit faculty will be able to familiarize themselves with the development process of remote laboratories, acquaint themselves with the Labshare project and access the tools required to build and implement their own remote laboratory.

\section{THE LABSHARE FAQ}

When initially being confronted with the idea of remote laboratories, there are numerous questions that are inevi- 


\section{A TOOLKIT FOR REMOTE LABORATORY DESIGN \& DEVELOPMENT}

tably asked, some more immediately than others. While dealing with individual colleagues it is possible to inform faculty on a case by case basis, however as the scale of the collaborations expand this becomes less practical. A listing of frequently asked questions enables this information to be accessed on demand. The FAQ, which has been evolving over approximately four years, currently includes information regarding the benefits of remote laboratories compared to other laboratory types, the advantages to staff and institutions, the purpose of LabShare and how to get involved. As remote laboratories mature and the scope of development shifts, the focus of questions will be updated to address the anticipated problems of the time. Some sample questions from Labshare's FAQ documentation are illustrated in figure 1.

\section{THE LABSHARE GLOSSARY}

A typical remote laboratory implementation requires a range of elements in order to be successful. The purpose of the glossary is to standardize the terms, and define the meanings, used to describe these reoccurring elements. A sample of Labshare's glossary is illustrated in figure 2.

Previously remote laboratory developers have worked in independent groups, developing their own prescribed terminology to describe important concepts. Given this trend it became prudent to develop and maintain a database that specifies the terminology used within the Labshare consortium. Currently included terms focus on describing the remote lab's physical rig, pedagogy, stakeholders and software.

An additional benefit of the glossary is that it enables faculty unfamiliar with remote laboratory terminology to adequately interpret the other information provided in the Labshare toolkit. As the toolkit expands over time to include a greater depth of information, the glossary will also continue to evolve, keeping Labshare members up to date in the developments of remote laboratories.

This research has considered not only the technical infrastructure that supports remote labs, but also the educational issues associated with their use. Consideration has been given to aspects such as: the way in which students engage with laboratories through a remote access modality; the impact of a lab being "real" vs "virtual" (or simulated); the effects on leaming outcomes; the types of labs that are best suited to remote access; etc. In other words, there is a solid research underpinning that validates the use of remote labs.

\section{Q: Why "Labshare"?}

Whilst support for improving student learning outcomes remains our primary driver, remote labs also enables the sharing of labs across multiple institutions. Historically, the geographic separation of labs made sharing impractical. With remote labs this is no longer an impediment, and so we can now consider how to support this sharing - and achieve the enormous benefits that can accrue if we support this sharing effectively.

The Labshare project is focussed on creatingthe technical and organisational frameworks that will enable this sharing to occur. The project mission states this best:

Labshare's mission is to create a nationally shared network of remote laboratories that will result in higher quality labs that support greater studentflexibility and improved educational outcomes, improved financial sustainability, enhanced scalability in terms of coping with student loads, and are developed and run by those with the best expertise."

\section{Q: Does the Labshare Project aim to replace hands-on labs with remote labs?}

No! We are aiming to provide an environmentfor sharing of remote labs in the circumstances where their use is appropriate, and where benefits can be gained throughtheir sharing. In some cases these shared labs will be a useful adjunct to existing facilities, and in other cases it may be appropriate for existing hands-on labs to be migrated to a remote access approach - but only where it is clear that doing so is educationally appropriate.

\section{Q: How will Labshare work?}

We don't know yet! Determining an appropriate model to supportlab sharing is one of the key objectives of the project. It may be that the right model is a central clearing house that institutions can subscribeto, and which provides both technical support and a brokerage service. Alternatively, it may be that institutions become share-holding members of a consortium that coordinates the sharing activities.

Figure 1. Example questions from Labshare's FAQ 


\begin{tabular}{|c|c|c|c|c|c|}
\hline Term $^{*}$ & Short term & \multirow{2}{*}{\multicolumn{4}{|c|}{ Definition ${ }^{\star}$}} \\
\hline Coldfire & none & & & & Master Server - Hosts the arbitration system and web interface. (Controls which users are allowed to access which experiment and for what tin \\
\hline control server & none & \multicolumn{3}{|l|}{ Provides access control for a rig and hosts the rig control software. (Usually virtual machines) } & \\
\hline master senver & master & \multicolumn{3}{|c|}{ Master Sever - Hosts the arbitration system and web interface. (Controls which users are allowed to access which experiment and for what time } & \\
\hline monitor server & none & \multicolumn{4}{|c|}{ Hosts audio-visual devices to 'monitor' or view the rigs. (Also provides a convenient place to host control sevvers to aggregate multiple rigs to one physical server.) } \\
\hline remote server & none & TBA & & & \\
\hline Access Control List & acl & \multicolumn{2}{|c|}{ list of permissions attached to an object } & & \\
\hline activity catalogue & catalogue & \multirow{2}{*}{\multicolumn{3}{|c|}{$\begin{array}{l}\text { A tagged, browsable, searchable view of all available Activity Designs intended to advertise the availability of Activity Designs } \\
\text { interface that a users sees or uses while carrying out an activity }\end{array}$}} & \\
\hline activity UI & none & & & & \\
\hline Batch exerciser & none & \multicolumn{3}{|c|}{ A rig specific control application allowing input of control and data files and the output of a result file following scheduled processing by rig device. } & \\
\hline Demonstration exerciser & none & \multirow{2}{*}{\multicolumn{3}{|c|}{ A rig specific control application designed to simulate interactive use by a human user by running a predefined sequence of commands }} & \\
\hline Laboratory Device & device & & & & \\
\hline laboratory rig collection & collection & \multicolumn{2}{|c|}{ The collection of homogeneous rigs, all of the same rig type, which are co-located and managed together. } & & \\
\hline laboratory rig configuration & rig config & & \\
\hline & & \multirow{2}{*}{\multicolumn{4}{|c|}{$\begin{array}{l}\text { A single instance of a physical system made from various devices (including associated software and hardware) that can be used by one or more students in carrying out } \\
\text { The collection of homogeneous rigs, all of the same rig type, which may be distributed across multiple physical locations and managed by different organisations, but whic }\end{array}$}} \\
\hline laboratory rig pool & pool & & & & \\
\hline laboratory rig type & rig type & \multicolumn{3}{|l|}{ The class to which a rig belongs, and within which any rig can be used interchangeably. } & \\
\hline physical hardware laboratory & y remote lab & \multirow{2}{*}{\multicolumn{4}{|c|}{$\begin{array}{l}\text { Multiple collections of heterogeneous rigs, possibly belonging to multiple rig types, which are co-located and managed together. } \\
\text { A complete single collection, single rig remote lab which can be shipped to a provider site. One of the } 3 \text { technology transfer mechanisms to be used for the sharing trials. }\end{array}$}} \\
\hline Remote lab in a crate & none & & & & \\
\hline Rig batch mode & Batch mode & \multicolumn{4}{|c|}{$\begin{array}{l}\text { A complete single collection, single rig remote lab which can be shipped to a provider site. One of the } 3 \text { technology transfer mechanisms to be used for the sharing trials. } \\
\text { Operating mode in which a rig in which a human user submits a set of parameters to be run against a rig by an automated exerciser. }\end{array}$} \\
\hline rig controller & none & \multicolumn{4}{|c|}{$\begin{array}{l}\text { Operating mode in which a rig in which a human user submits a set of parameters to be run against a rig by an automated exerciser. } \\
\text { device that controls or runs the rig. }\end{array}$} \\
\hline Rig demonstration mode & Demonstration mode & \multicolumn{4}{|c|}{ Operating mode in which interactive use of a rig by a a human user rig is simulated by an automated exerciser for marketing purposes. } \\
\hline Rig Exerciser & Exerciser & \multicolumn{4}{|c|}{ A rig specific control application run against a rig to automate a particular function. Rig exerciser types include batch exercisers, demonstration exercisers and system gi } \\
\hline Rig interactive mode & Interactive mode & \\
\hline rig monitor & none & \multicolumn{4}{|c|}{ monitors the rig and provides rig status (i.e rig good, rig bad, rig fault ) back to the system. Monitors the rig and the rig controller. } \\
\hline Rig operating Mode & mode & \multirow{2}{*}{\multicolumn{4}{|c|}{$\begin{array}{l}\text { One of the } 3 \text { ways that a rig can be used operationally, the modes are interactive, batch and demonstration. } \\
\text { A rig specific control application designed to test rig function by running a predefined sequence of commands and comparing outpu }\end{array}$}} \\
\hline SystemGood exerciser & none & & & & \\
\hline SystemGood flag & none & \multicolumn{4}{|c|}{$\begin{array}{l}\text { A rig specific control application designed to test rig function by running a predefined sequence of commands and comparing outpu } \\
\text { The mechanism by which the SystemGood exerciser communicates to the arbitrator that a particular rig is available for allocation }\end{array}$} \\
\hline Consortium Product & none & \multicolumn{4}{|c|}{ The mechanism by which the SystemGood exerciser communicates to the arbitrator that a particular rig is available for allocation } \\
\hline Consumer & none & \multicolumn{4}{|l|}{$\begin{array}{l}\text { Ane of the two products of the consootium le what Is traded nigs and activty designs } \\
\text { An institutional consumer of consortium products. }\end{array}$} \\
\hline Consumer/Provider & none & \multicolumn{4}{|l|}{ An institutional entity which both uses and consimes consortium products } \\
\hline Provider & none & \multirow{2}{*}{\multicolumn{4}{|c|}{$\begin{array}{l}\text { An institutional provider of consortium products. } \\
\text { An institutional consumer of consortium products who does not also provider consortium products }\end{array}$}} \\
\hline Pure Consumer & none & & & & \\
\hline Pure Provider & none & An institutional provider of consortium products who does not also consume consortiur & & & \\
\hline guarranteed lease & none & Provision of resources is guaranteed for the term of the lease. Access is exclusive to $t$ & the lease holder & & \\
\hline lease & lease & A lease is an agreement between a provider and a consumer to provide access to a de & defined set of resources (eg a Rig). L & Leases have a valid time period in $\mathrm{w}$ & ih they can \\
\hline market & market & A entity that can be used to trade leases & & & \\
\hline
\end{tabular}

Figure 2. Sample of Labshare's glossary component of the toolkit

\section{LITERATURE REVIEW}

Remote laboratories first appeared in the Engineering Education literature in the mid 1990's [9]; since that time there have been some well-known and well-understood themes that have emerged in the field. The toolkit seeks to provide developers with access to this literature, supporting them both during their early orientation to remote laboratories, and as they seek to expand their expertise.

Labshare's current endnote library includes over 380 references from a wide variety of authors. This comprehensive index of the field is intended to assist faculty in exploring aspects of the field of remote laboratories in great depth by guiding them to relevant articles within the field.

Labshare has comprised listings of “Top Ten” papers for categories that will inform faculty of the trend in development for remote laboratories. These listing include informative papers for introducing faculty with no remote laboratory experience and listings of the most cited papers to date. When viewed in their entirety the introductory papers provide a sufficient picture of the role of remote laboratories in engineering education. Information provided includes, but is not limited to, prominent remote laboratory disseminations, in depth comparisons between access modes and examples of remote laboratory deployment and development processes.

Research into the top ten most cited papers was also conducted using various sourcing tools, including Google Scholar, ISI Web of Knowledge and Scopus. These rankings are updated regularly and are also available for viewing on the Labshare website. This is a reasonable indication of the most widely accessed academic papers in the field of remote laboratories, and as such shows the prominent areas of development being followed by the majority of faculty.

\section{A FRAMEWORK FOR DETERMINING Rig SUITABILITY}

Before commencing a remote laboratory implementation, it is essential to determine whether the laboratory experience is in fact suitable for remote operation. This segment of the toolkit provides the user with a framework to assess this suitability. Factors taken into account by the framework include learning factors, cohort factors and equipment factors. A segment of Labshare's framework document is showcased in figure 3 .

There are various laboratory characteristics which encourage or discourage a conversion to enable remote access. These include information flow, measurement methods, collaborative, supervisory, accessibility and health and safety.

Given the nature of remote labs, such as round the clock accessibility and repeated use, recognizable forms of lab equipment are of a less compatible nature than others. The appropriateness of lab equipment includes the cost to run, if consumables are used regularly, the reproducibility of results and the availability of equipment.

The characteristics of the student body making use of the lab will also play a vital role in assessing the suitability of a remote lab conversion. Ease of internet access, geographical distribution and the student to rig ratio are

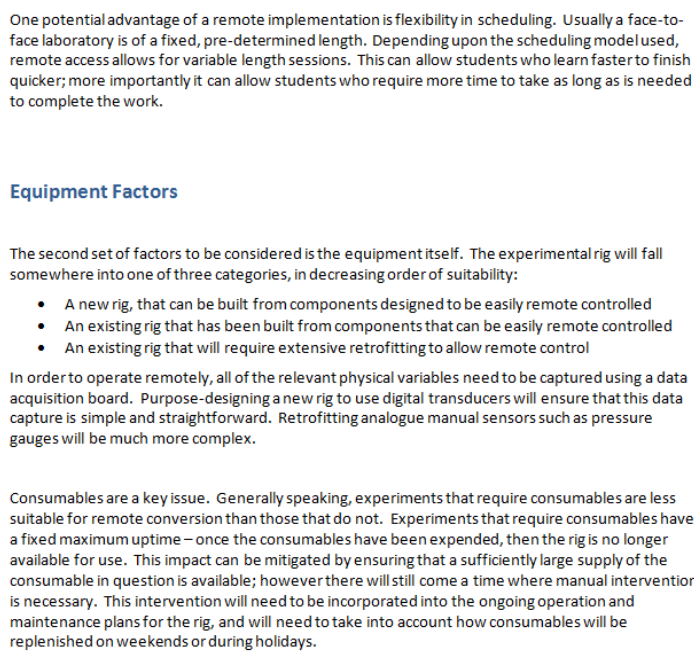

Consumables are a key issue. Generally speaking, experiments that require consumables are less suitable for remote conversion than those that do not. Experiments that require consumables have a fixed maximum uptime - once the consumables have been expended, then the rig is no longer available for use. This impact can be mitigated by ensuring that a sufficiently large supply of the consumable in question is available; however there will still come a time where manual interventio is necessary. This intervention will need to be incorporated into the ongoing operation and maintenance plans for the rig, and will need to take into account how consumables will be replenished on weekends or during holidays.

Figure 3. section of equipment factors influencing a rig's suitability 
examples of factors that influence the appropriateness of a hands-on to remote lab conversion.

This part of the toolkit is substantially based upon [10].

\section{A SNAPSHOT OF THE LABShare CATALOGUE}

In order to keep track of the remote laboratories that are under development, as well as those already available, a catalogue that showcases the rigs that comprise the Labshare network is available online. Each listing includes detailed information including availability, access modes, rig type, target discipline and year, a description of the experiment and the provider institution and academics. An example of a remote laboratory included in Labshare's catalogue is shown in figure 4.

Currently the Labshare toolkit includes a snapshot of the catalogue to enable distribution in an offline capacity. It is planned to convert the catalogue into an online searchable database.

\section{ACCREDITATION COMMENTARY}

One of the most commonly expressed fears regarding remote laboratories is whether accreditation bodies will consider them to be acceptable. The toolkit addresses these concerns by providing a guide to incorporating remote laboratories into the responses to the accreditation criteria, both for ABET and for Engineers Australia.

Accreditation reviews, of relatively new developed technologies, is of particular interest to academics interested in developing and implementing their own courses. Without the support of the wider community, it is potentially difficult to cater a lesson plan to specific criteria that accreditation bodies require. However given the support Labshare provides, in the form of an accreditation commentary, new members are able to start developing immediately.

Each of the accreditation criteria are addressed in turn and are categorized as directly relevant, marginally relevant or irrelevant to remote laboratories. For each criterion that is considered relevant, guidance is given for explaining how remote laboratories contribute to the outcomes of the overall degree program, and how they can be implemented without jeopardizing the overall quality of the program's graduates.

\subsection{Mapping, Localisation and Obstacle avoidance}

Audience: $2^{\text {nd }}$ to final year university students

Discipline: Mechanical Engineering, Mechatronics Engineering

Status: Available

Provider Institution: UTS

Lesson Author: Dr Sarath Kodagoda

Abstract: Using Bayers rule and the onboard sensors students have to program the robot to carry out mapping then localise the robot on the map using particle filters and Kalman filtering. The robot then has to patrol the generated map avoiding any present obstacles using path planning.

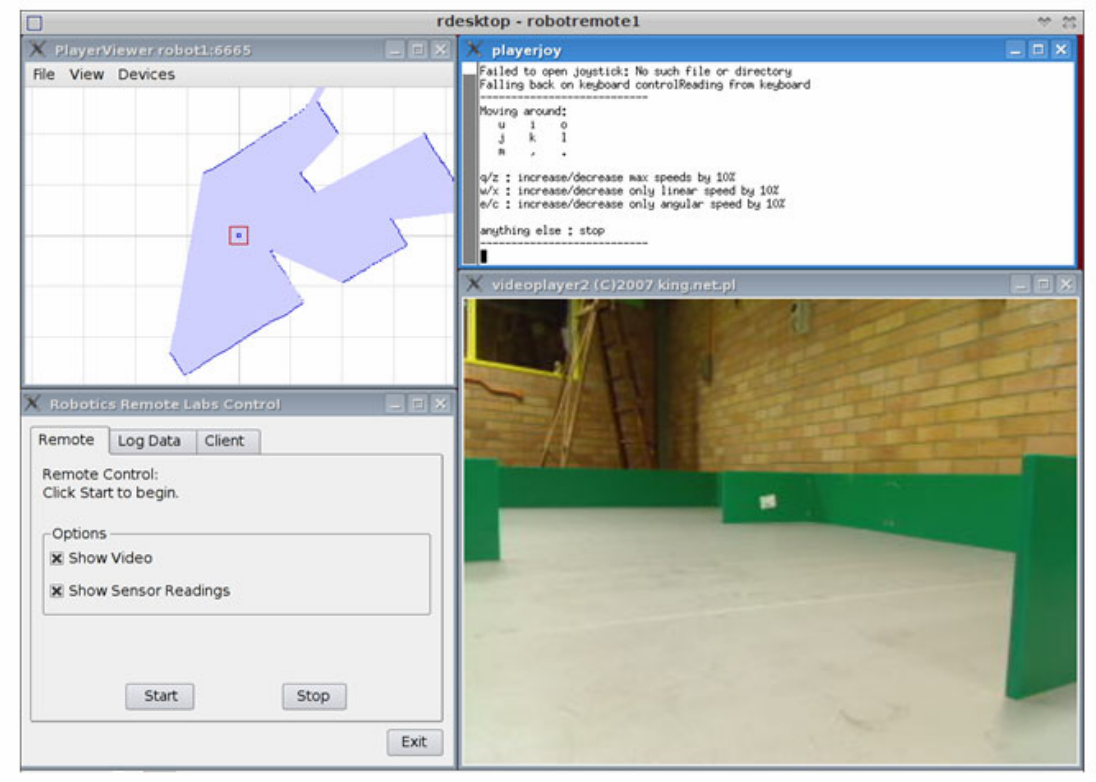

Figure 4. example of a remote lab included in labshare's catalogue 
A well-designed and well-implemented remote laboratory may in fact be more educationally beneficial for students than the traditional face to face laboratory it is supplementing or replacing. The laboratory needs to be considered in the context of the overall degree program, and presented to the accreditation panel as part of the overall education of the engineering students. This part of the toolkit provides advice on how best to do this presentation.

\section{VIII.LESSON PLANS}

The lesson plan portion of the toolkit provides a standardized template that can be used for a remote laboratory setup. Given the essential focus of ensuring students a high quality learning experience, the educational design of laboratory classes is considered an exceptionally important part of participating in Labshare.

The current version of the template is considered comprehensive enough to put into practice and as such has been used to create a variety of lesson plans for existing laboratories. Key components of the lesson plan include an overview, goals, prerequisites, rig information and assessment information.

To ensure the quality of lessons provided by Labshare, lesson templates require a significant investment of time and research to complete. Therefore in order to assist users in the task, exemplar lesson plans are provided. Currently examples include loaded structural beam, PLC programming, determination of gravitation acceleration and structural visualization remote laboratories. As the lesson plans becomes more widely accessed additional assistance will be provided if necessary.

\section{Evaluation Questions}

When implementing any major change, it is important to evaluate its effectiveness. Labshare implementation covers a range of different changes, and as such it is important to have access to a range of evaluation tools. The Labshare toolkit contains instruments to survey the development, deployment and learning attributes of potential remote laboratory setups. Each survey poses a series of questions, which enables an analysis of components that will make successful implementation of a remote lab rig more likely. Also included in the toolkit are approximately 150 additional questions, which will allow users to build their own assessment tools as required. Example questions from documentation provided in Labshare's toolkit are shown in figure 5.

\section{Ongoing DEVElopment}

The Labshare project is currently carrying out evaluation and sharing trials. Throughout the second semester of 2010 and first semester 2011, a total of over four thousand students from approximately nine institutions will be using Labshare-administered remotely accessible experiments in support of their learning. This undertaking has been carefully planned to ensure that participation in the trials incorporates not just use of some experimental apparatus over the Internet, but that the whole user experience is carefully assessed. Student users are requested to complete an online survey after finishing their learning exercise and the teaching staff are asked to provide a summary of their impressions of the experience. It is anticipated that the large volume of data gathered throughout this exercise

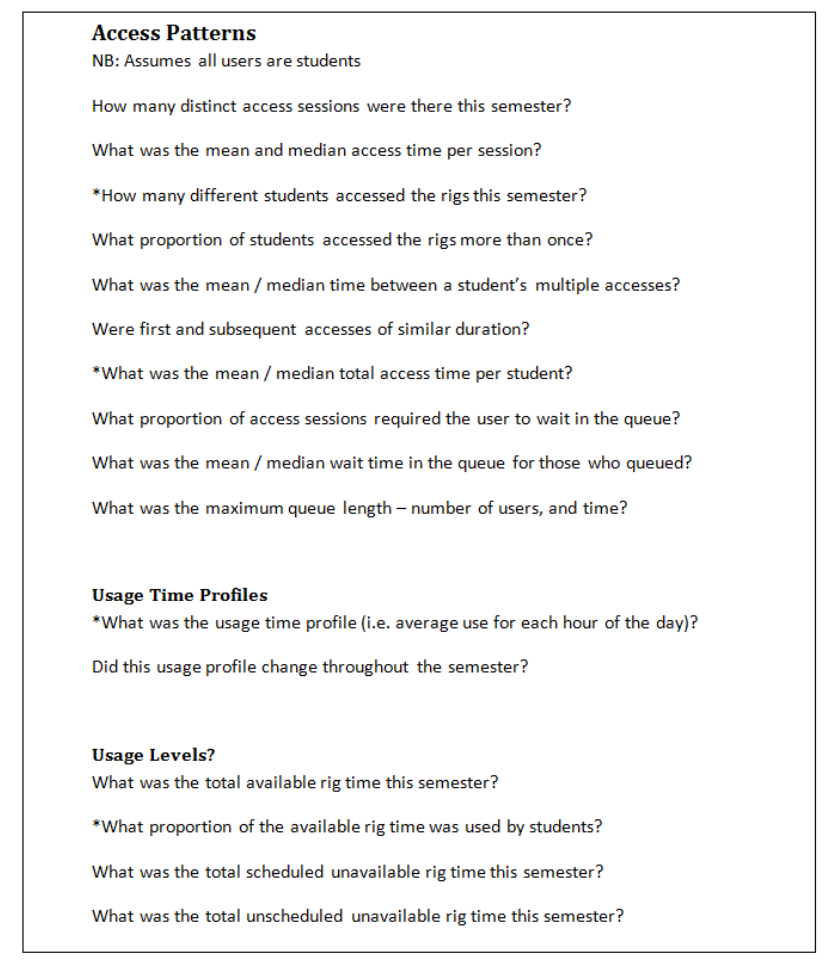

Figure 5. Evaluation questions from Labshare's evaluation survey

will present several avenues of continued development which will strengthen the toolkit considerably.

\section{CONCLUSION}

By providing the presented toolkit to new, existing and potential members of the Labshare consortium, the information and planning required to produce high quality remote laboratories has become freely available. Not only does this present an easily accessible repository to faculty current specializing in remote laboratories, it also paves the way for faculty unfamiliar with internet-based control to become involved in the development process. By expanding the pool of potential participants actively involved in the development process, benefits such as the pooling of resources and increasing student accessibility to a diverse range of experimentation will become more fully realized.

\section{REFERENCES}

[1] V. Harward, et al., "The iLab shared architecture: A Web Services infrastructure to build communities of Internet accessible laboratories," Proceedings of the IEEE, vol. 96, pp. 931-950, 2008. http://dx.doi.org/10.1109/JPROC.2008.921607

[2] I. Gustavsson, et al., "The VISIR project an Open Source Software Initiative for Distributed Online Laboratories," REV 2007, 2007.

[3] T. Richter, et al., "LiLa: A European Project on Networked Experiments," in REV 2009, 2009.

[4] D. Lowe, et al., "LabShare: Towards a National Approach to Laboratory Sharing," in AAEE 2009, Adelaide, Australia, 2009.

[5] B. Aktan, et al., "Distance learning applied to control engineering laboratories.," IEEE Transactions on Education, vol. 39, pp. 320326, 1996. http://dx.doi.org/10.1109/13.538754

[6] E. D. Lindsay and M. C. Good, "Virtual and Distance Experiments: Pedagogical Alternatives, not Logistical Alternatives," in ASEE 2006: Annual Conference \& Exposition, Chicago, Illinois, 2006, pp. 19-21.

[7] S. Esche, "Remote experimentation - one building block in online engineering education," in ASEE/SEFI/TUB 2002 Colluquium, 2002. 
[8] J. Henry, "Controls laboratory teaching via the World Wide Web," 1996.

[9] E. Lindsay, et al., "Derivation of Suitability Metrics for Remote Access Mode Experiments," in REV 2010, Stockholm, Sweden, 2010.

\section{Authors}

E. D. Lindsay is an Associate Professor at the School of Civil \& Mechanical Engineering at Curtin University, Perth, Australia (email: e.lindsay@curtin.edu.au)

S. Murray, is a Senior Lecturer at the Faculty of Engineering \& Information Technology at the University of Technology Sydney, Sydney Australia (e-mail: stevem@eng.uts.edu.au).

B. D. Stumpers is a Research Associate at the School of Civil \& Mechanical Engineering at Curtin University, Perth, Australia (email: B_Stumpers@curtin.edu.au)

This work was supported by the Department of Education, Employment and Workplace Relations (DEEWR)'s Diversity and Structural Adjustment Fund. It is an extended version of a paper presented at the GOLC remote Laboratories Workshop, held October 2011, in Rapid City, South Dakota, USA. Received 15 November 2011, 2011. Published as resubmitted by the authors 9 February 2012 